\title{
Comparative study of Metal Antenna \&Plasma Antenna
}

\author{
Pramod Bhavarthe $^{1}$, Leena Govekar ${ }^{2}$, Priti Tyagi ${ }^{3}$ \\ Assistnat Professor, Electronics, PVPPCOE, Mumbai, India ${ }^{1,2}$ \\ Associate Professor, Electronics, PVPPCOE, Mumbai, India ${ }^{3}$
}

\begin{abstract}
Metal antennas currently in implementation use metallic conductor as guiding medium for electromagnetic Radiations. Plasma antennas uses ionized medium. The plasma antenna is a radiofrequency antenna formed by a plasma columns, filaments or sheets, which are excited by a surface wave. The relevance of this device is how rapidly it can be turned on and off only applying an electrical pulse. In this paper we have discussed the basic theory, operation of the plasma antenna. We have also given the features, advantages and applications for the same.
\end{abstract}

Keywords: Plasma Antenna, Plasma frequency, ionized gas, metal antenna

\section{INTRODUCTION}

Plasma antennas are radio frequency antennas that employ plasma as the guiding medium for electromagnetic radiation. Here, plasma discharge tubes are used as the antenna elements. The tubes become conductors when they are energised and can transmit and receive radio signals. And they revert to non-conducting elements when de-energized. In this state they not are probing radio signals. Plasma antenna can be "Steered" electronically. It can also be turned off rapidly reducing the effect of ringing on pulse on transmission[1].

In recent years, the rapid growth in both communications and RADAR systems has led to a concomitant growth in the possible applications and requirements of antennas. These new requirements include compactness and conformity, rapid configurability for directionality and frequency agility and for military applications should also allow low absolute or out-of-band radar cross-section and facilitate low probability of intercept communications. Investigations have recently begun on the use of ionized gases or plasmas as the conducting medium in antennas that could satisfy these requirements.

Such plasma antennas may even offer a viable alternative to metal in existing applications when overall technical requirements are considered. Possibilities of the plasma application for antenna parameters control have been proposed in the sixties of 20 century. In work the test data of $10 \mathrm{GHz}$ signal transmission are presented. The transmission was realized along a plasma channel that was created by the atmosphere magnetized plasma and the shortcut dipole girded breakdown. The atmosphere breakdown was created by the focused laser emission. Only a few works are known, for example, that carries results of calculations of electromagnetic field reflected by surface girded by plasma layer. It was experimental shown that an interaction of the by the plasma leads to increasing of the signal power emitted into a free space. But there is insufficient quantity of the works dedicated to development of the plasma antennas designed for work in the terrestrial atmosphere conditions. Methodical researches in that area have not done yet.[2]

\section{WORKING}

\section{A. Basic Theory and Plasma Parameters}

Plasma in terms of electromagnetic properties is a nonhomogenous, non-linear and dispersive environment. Permeability $(\mu)$, conductivity $(\sigma)$ and permittivity $(\varepsilon)$ in plasma can be varied in terms of frequency \& other parameters and make plasma a special environment.As a result, for any frequency of the incident wave and in any density of ionization, one particular response occurs.

Radiated electromagnetic waves on plasma will absorb, scatter or pass through. We can choose to absorb, scatter or pass through with changing the basic parameters like electron density and collision frequency. The relative permittivity of plasma is defined by [6]

$$
\varepsilon_{r}=\varepsilon_{r}^{\prime}-j \varepsilon_{r}^{\prime \prime}=1-\frac{w_{p}^{2}}{w(w-j v)}
$$

Where, $w_{\mathrm{p}}$ is plasma frequency, $\mathrm{w}$ is operating frequency and $\mathrm{v}$ is collision frequency. The plasma frequency is a measure of the amount of ionization in the plasma and the operating frequency of the plasma antenna is the same as the operating frequency of a metal antenna. Plasma frequency is equal to [ ]

$$
w_{p}=\sqrt{\frac{4 \pi n_{e} e^{2}}{m_{e}}}
$$

Where $\mathrm{n}_{\mathrm{e}}$ is electron density, $\mathrm{e}$ is the charge of electron and $m_{e}$ is the electron mass. The electron density is defined by [6]

$$
n_{e}=\frac{J}{e \sqrt{\frac{k T_{e}^{o}}{m_{e}}}}(3)
$$

Where $\mathrm{J}$ is current density, $\mathrm{k}$ is Boltzmann's constant and $\mathrm{T}_{\mathrm{e}}$ is electron temperature which is calculated for fluorescent lamps [6]

The boundary conditions at a dielectric-vacuum interface are that the normal component of the displacement and tangential component of the electric field be continuous. These conditions are satisfied at the plasma-air boundary. Satisfaction of the boundary conditions gives the potential inside the plasma in terms of the incident amplitude and frequency and it is shown that the field in the plasma becomes large (resonant) when the frequency is [6]: 
INTERNATIONAL JOURNAL OFINNOVATIVE RESEARCH IN ELECTRICAL, ELECTRONICS, INSTRUMENTATION AND CONTROL ENGINEERING Vol. 3, Issue 1, January 2015

$$
\omega=\frac{\omega_{p}}{\sqrt{2}}
$$

Since the plasma column is in resonance at (4), the electrons in the column oscillate in response to the driving electric field. This motion reradiates, or scatters, the incident field in cylindrical waves. Since the motion of the electrons in the plasma is largest at resonance, the scattered power will be a maximum at resonance.

In the case of $\omega>\omega_{p}$, propagation constant $(\gamma)$ as shown in (5) becomes imaginary and the wave will propagate. However, if $<\omega_{p}$, the propagation constant becomes real and wave will not propagate [6]

$$
\gamma=\alpha+j \beta=j k_{0} \sqrt{\mu_{r} \varepsilon_{r}}
$$

\section{B. Plasma Antenna}

Plasma antenna technology employs ionized gas enclosed in a tube (or other enclosure) as the conducting element of an antenna [1,4]. This is a fundamental change from traditional antenna design that generally employs solid metal wires as the conducting element. Ionized gas (plasma) is an efficient conducting element with a number of important advantages [3]. The design allows for extremely short pulses, important to many forms of digital communication and radars [1].

One fundamental distinguishing feature of a plasma antenna is that the gas ionizing process can manipulate resistance [3]. A second fundamental distinguishing feature is that after sending a pulse the plasma antenna can be deionized, eliminating the ringing associated with traditional metal elements [3]. Ringing and the associated noise of a metal antenna can severely limit capabilities in high frequency short pulse transmissions.

When voltage applied to an antenna, electric field is produced and this electric field causes current to flow in the antenna [3]. Due to current flow, magnetic field is then produced. These two fields are emitted from an antenna and propagate through space over very long distances. The applications of plasma antenna is in high speed digitalcommunication and radar system, radio antenna, stealth for military application and can be used for transmission and modulation techniques (PM, AM, FM) [2]. The advantages of plasma antenna are in its high power, enhanced bandwidth, higher efficiency, lower thermal noise, perfect reflector, low in weight, smaller in size, and improved reliability [4].

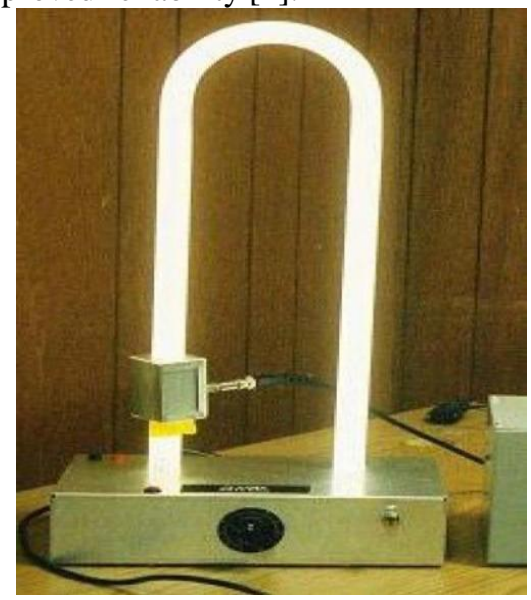

Fig. 1: Plasma Antenna 
replacing metal conductors with semi-conductor chips or gases

iv) Dynamically reconfigurable: The gain, frequency, and polarization, power, etc. of the plasma antenna can be changed by the handler of the antenna. A single plasma antenna can do work of multiple normal antennas thus reducing the space and cost required for operation.

v) Fast Transmission: By using plasma semiconductor antenna the electromagnetic waves produced can be focused to form a beam which travels faster than wave when certain diodes are activated.

\section{F. Disadvantages}

i) Complex Design: Use of plasma makes the design of antenna complex.

ii) Cannot Penetrate Walls: The semi-conductor plasma antenna developed by the inventors was limited to high frequency hence their use also gets limited. Also the signal from these antennas couldn't penetrate the walls. This can resolved by using reflectors to make waves reach the destination.

iii) More Power: More power is used to ionize the gas; hence ionizer increases power consumption of the antenna. iv) Stable and repeatable plasma volumes: Not all of the gas is ionized to become plasma, some parts remain unionized. Thus the volume of the plasma formed during each time should be same to generate stable electromagnetic waves. This can be achieved by keeping the current flowing through it constant, which will excite the same amount of particles.

Application

Plasma antennas find its applications in variety of fields due its unique properties, characteristics and advantages over traditional metallic antennas.

i)Radio and television broadcasting: Signal strength of signals emitted by plasma antennas is relatively stronger than traditionally used normal metal antennas hence it lasts longer without damping and being extinguished hence broadcasting companies may require less relay stations and repeaters to relay signals to further areas and hence it may bring down cost of the broadcasting system for service provider companies.

ii) Space communications: Plasma antennas are relatively lighter than normal antennas and hence can used as communication devices in spacecraft's like jet planes, commercial planes, even in space shuttles and also in unmanned air vehicle sensor antennas.

iii) Military applications: Invisibility to radar means that signals sent by plasma antennas are very difficult to be detected by any outsider and as military needs to send and receive top secret information without any outside interception plasma antennas have qualities and potential that can be used to develop and produce equipment's in military applications within foreseeable future

iv)Faster internet: Plasma antennas can provide a faster rate of data transmission and hence can be used to provide high speed internet like Wi-Gig (Wireless Gigabit Alliance) whichis faster than Wi-Fi.

v) Public safety networks: Public safety networks like CCTVs are used to prevent crimes, track down criminals and also may be accepted as video evidence in court. If these devices are tampered or damaged by criminals then it is possible to for a handler from safety department to diverge or reroute the traffic using plasma antennas.

\section{III.CONCLUSION}

The principle behind the working of the plasma antenna is same as the normal conventional antennas. Only the solid metal conductor is replaced with the plasma. This plasma gives it many advantages over the current antennas. It is more efficient, fast and also can be manufactured cheaply. There might be some disadvantages associated with plasma antennas but those can be overcome. It will take some time for plasma antennas to be commercially available, but it will change the landscape of antennas when it is available for use.

\section{REFERENCES}

[1] P.Darvish, BijanZakeriDesign,Simulation And Implementation of Pre-ionized Coupled Plasma Antenna at VHF Band, Proceedings of 'ISET', 2013.

[2] Alexeff,T. Anderson, S.Parameswaran, E.Prdeep, Experimental and theoretical results with plasma antennas, , IEEE Transactions on plasma Science Vol.34, No.2,2006

[3] H.Q.Ye, M.Geo and Chang J, Tang, "Radiation theory of the plasma antenna ," IEEE Transactions on antennas \& propagation, Vol.59,No.5, May-2011

[4] W.Jiayin, Sh. Jiaming, W.Jiachun, Xu., "study of the radiation pattern of the unipole plasma antenna”, IEEE conf, ISAPE, International Symposium

[5] RitikaNahar, ShaifaliTiwari, GarimaTiwari, "Plasma Antenna", IJETMER, vol.1, issue 1, feb-13

[6] C. Patel, N. Nasani, T. Parekh, "Plasma Antenna”, IJETT, vol.15 no.6 sep-14Books

[7] Theodore Anderson, "plasma antennas",Artechhouse, 2011 